\title{
Repetitive afferent electrical stimulation of the lower-limb: effect on corticomotor excitability and implications for rehabilitation
}

\author{
Guillaume Léonard $^{1,2}$, Catherine Mercier ${ }^{1}$, Louis E. Tremblay ${ }^{3}$ \\ ${ }^{1}$ Centre interdisciplinaire de recherche en réadaptation et en intégration sociale (CIRRIS) de \\ l'Université Laval, 585 boulevard Hamel, Québec (Québec), Canada, G1M 2S8; ${ }^{2}$ École de \\ réadaptation de l'Université de Sherbrooke, $300112^{\mathrm{e}}$ avenue Nord, Sherbrooke (Québec), \\ Canada, J1H 5N4; ${ }^{3}$ Département des sciences de la santé de l'Université du Québec à Chicoutimi, \\ 555 boulevard de l'Université, Chicoutimi (Québec), Canada, G7H 2B1
}

Corresponding author: Guillaume Léonard, $\mathrm{Ph} . \mathrm{D}$.

École de réadaptation

Faculté de médecine et des sciences de la santé

Université de Sherbrooke

3001, 12e avenue Nord

Sherbrooke (Québec) J1H 5N4

Sources of support: Guillaume Léonard and Catherine Mercier are supported by the Canadian Institutes of Health Research. 


\begin{abstract}
Despite the extensive work published on the effects of electrical stimulation on corticomotor excitability, very few studies have focused on lower limb muscles. The aim of the present study was to determine the effect of high-frequency afferent electrical stimulation of the anterior thigh area on the corticomotor excitability of lower limb muscles. Twenty-two healthy subjects (mean age $23 \pm 7 \mathrm{yrs}$ ) participated in the study. Electrical stimulation was applied for 60 minutes on the anterior thigh area (frequency $100 \mathrm{~Hz}$, pulse duration $60 \mu \mathrm{s}$, intensity below motor threshold). Motor evoked potentials (MEP) of the rectus femoris and biceps femoris muscles were recorded before, and after the electrical stimulation paradigm with a Magstim 200 stimulator. Analyses revealed a significant modulation in MEP amplitude for the RF but not for the BF muscle. Specifically, there was a significant reduction in MEP amplitude for the RF muscle immediately, 15 minutes and 30 minutes after the end of electrical stimulation when compared with baseline. The present results indicate that a 60 -minute high-frequency electrical stimulation protocol applied on the anterior thigh area decreases the corticomotor excitability of the RF muscle. Although the exact duration remains unknown, the pattern of modulation observed indicates that the inhibitory effect lasts for more than 30 minutes after the end of stimulation, giving enough time for clinicians to work on the desired motor task during rehabilitation.
\end{abstract}

Keywords: $\quad$ Transcutaneous electrical nerve stimulation (TENS), transcranial magnetic stimulation (TMS), motor evoked potential (MEP), rehabilitation 


\section{Introduction}

Neurons of the central nervous system have the ability to modify their connections and alter their functional organization, a phenomenon called plasticity $(1,2)$. Apart from being important from a neurophysiological perspective, principles of neural plasticity bear important considerations for rehabilitation professionals working with people suffering from diverse neurological conditions. In fact, the ability of the nervous system to reorganize itself following an insult is the fundamental principle underlying the rehabilitation of many neurological conditions such as stroke and traumatic brain injury (3).

Several techniques can be used to promote and optimize plasticity, including behavioural training, functional exercises and sensorimotor relearning techniques (4-6). In 1990, Recanzone et al. (7) demonstrated that repetitive electrical afferent stimulation could also be used to induce plastic changes in the nervous system. They showed that the application of repetitive electrical nerve stimulation over the dorsal cutaneous branch of the ulnar nerve of felines increased the receptive fields of the primary somatosensory cortex (S1) associated with the ulnar nerve territory. These observations were followed by several transcranial magnetic stimulation (TMS) studies which showed that prolonged peripheral electrical stimulation could modulate the corticospinal excitability of human subjects and induce transient changes in cortical representation (8-12).

Interestingly, the nature of the modulation induced by electrical nerve stimulation appears to be highly dependent upon the different stimulation parameters used. For instance, low-frequency stimulation protocols have been known to increase corticospinal excitability $(8,9)$, whereas less consistent results have been obtained for high-frequency stimulation protocols, with some authors reporting increased (13), decreased (10) or unchanged (8) motor-evoked potential (MEP) responses. Another important parameter which is likely to influence the nature of the modulation is the site of stimulation per se. Given the functional importance of the hand for the human species, and its unique 
representation in the sensorimotor cortex, one cannot simply assume that the changes occurring after stimulation of this area will be the same as results obtained when stimulating the thigh or the foot. Unfortunately, the vast majority of TMS studies focusing on the effects of repetitive afferent electrical stimulation have mainly concentrated on hand and pharyngeal muscles (8-12), and very few have sought to determine the effect of afferent repetitive electrical nerve stimulation on the corticospinal excitability of lower-limb muscles (13). The aim of the present study was to address this issue by investigating the effect of a prolonged high-frequency electrical nerve stimulation protocol applied over the anterior thigh area.

\section{Methods}

\section{Participants}

Twenty-two male subjects (mean age $23 \pm 7 \mathrm{yrs}$ ) participated in the study. All participants were considered healthy at the time of testing (no neurological or musculoskeletal impairments based on self-report) and none presented contraindications to TMS. The Institutional Review Ethics Board approved the study procedure and written informed consent was obtained before the experimental session.

\section{TMS and recording of MEPS}

TMS was administered with participants seated in a comfortable chair. Magnetic stimulation was produced using a Magstim 200 (Magstim Co. Dyfed, UK) connected to a $90 \mathrm{~mm}$ double-cone coil. Small auto-adhesive surface electrodes (Ag-AgCl) were placed over the motor point of the rectus femoris (RF) of the participants' dominant lower extremity. Surface electrodes were also placed over the motor point of the biceps femoris (BF) in six participants in order to determine the specificity of MEP changes. The electromyographic (EMG) signals were amplified and filtered (bandwidth, $10 \mathrm{~Hz}$ to 1 $\mathrm{kHz}$ ) with a polygraph amplifier (RMP-6004, Nihon-Kohden Corp.). EMG signals were 
digitized at a $2 \mathrm{kHz}$ sampling rate using a custom software running under Microsoft Windows and equipped with a digital/ analogue acquisition card (BNC-2090, National Instrument Corp, Austin, TX, USA).

To determine the optimal site to evoke MEPs, participants were fitted with a Lycra swim cap with marking grids traced onto it. Head movements were restrained during testing using a U-shape cushion, which was fitted at the neck of the participant. With the stimulator set to $75 \%$ of maximal stimulator output, the approximate location of the thigh area on the contralateral primary motor cortex (M1) was explored in 1-cm steps until reliable MEPs could be evoked in the RF. This site (hotspot) was then marked with a red dot to ensure consistent coil positioning by the experimenter who frequently reassessed the coil position to ensure that it remained over the optimal stimulation site throughout the experiment. Following this procedure, the resting motor threshold (rMT) was determined using the method advocated by Mills and Nithi (14). Starting from suprathreshold intensity, the stimulator's output was gradually decreased by $1 \%$ steps until no MEPs could be evoked for ten consecutive stimuli. This TMS intensity corresponded to the lower threshold value. From this point, the intensity was gradually increased until MEPs of at least $50 \mu \mathrm{V}$ in peak-to-peak amplitude could be evoked in ten consecutive stimuli. The latter intensity determined the upper threshold value. The rMT was defined for each participant as the median intensity between the upper and lower threshold values. The TMS intensity was then fixed at the rMT $+10 \%$ of MSO for the remainder of the experiment. At this supra-threshold intensity, reliable MEPs could be evoked in the $\mathrm{RF}$ and BF muscles.

\section{Baseline MEP values}

Prior to the application of the electrical stimulation, MEPs were recorded at rest to establish baseline values. For these recordings and the remainder of the experiment, subjects were instructed to remain immobile and relaxed. EMG silence was verified 
visually on an oscilloscope at high gain amplification. With the subjects at rest, ten MEPs were recorded, with at least five seconds between stimulations.

\section{Electrical stimulation protocol}

Once the baseline values for MEPs were established, repetitive afferent stimulation was applied on the anterior thigh area (L2-L3 dermatomes) for 60 minutes using a transcutaneous electrical nerve stimulation (TENS) apparatus (Duo 500, Gymna, Netherlands) connected to flexible carbon electrodes $(5 \times 7 \mathrm{~cm})$. With the frequency set at $100 \mathrm{~Hz}$ and pulse duration at $60 \mu \mathrm{s}$, the stimulator's intensity was gradually increased and adjusted to produce a strong comfortable tingling sensation without inducing muscle activity. MEPs were recorded immediately after TENS application, as well as 15 and 30 minutes after the end of the stimulation period.

\section{Data analysis}

The peak-to-peak amplitude of MEPs recorded in each condition was measured offline and averaged for each participant to derive mean values. Because MEP amplitude data were not normally distributed, individual mean values were transformed into natural logarithms, as suggested by Nielsen (15). Following this transformation, visual inspection and Shapiro-Wilk tests indicated that the MEP amplitude data for the RF was distributed normally (all p-values $>.10$ ). Repeated-measures analysis of variance (ANOVA) were then performed to compare the MEP log-amplitude of the RF between the different time measures (baseline, 60 minutes, 75 minutes and 90 minutes). Significant results were followed with paired sample t-tests comparing each post-stimulation measure with baseline. Because of the small number of participants included in the BF analyses $(n=6)$ and since visual inspection revealed that the data for the BF was still not normally distributed after log-transformations, the analyses for the BF muscle were performed using non-parametric equivalents (i.e. Friedman's and Wilcoxon Signed-Rank tests). 
Differences were considered to be significant if $p<0.05$ was obtained. All tests were performed using SPSS version 18.0 for Windows (Chicago, IL, USA) and figures were prepared using GraphPad Prism version 5.03 for Windows (San Diego, CA, USA).

\section{Results}

The mean stimulation intensity for TENS was $54.9 \pm 13.6 \mathrm{~mA}$. This intensity corresponds to approximately twice the mean TENS perceptual threshold (30.6 \pm 13.6$)$. All participants reported the stimulation to be comfortable and non-painful.

The average MEP log-amplitude for the RF and BF obtained at baseline, 60, 75 and 90 minutes is presented in Figure 1a and 1b. As can be seen from these figures, there was a significant modulation of the MEP log-amplitude for the RF but not for the BF. The ANOVA confirmed the significant change in MEP log-amplitude across the different time measures for the RF $(p<.001)$. Post-hoc paired sample t-tests revealed a significant reduction in MEP log-amplitude at 60,75 and 90 minutes when compared with baseline (all p-values $<.05$ ), indicating that the high-frequency TENS protocol significantly reduced the corticospinal excitability of the RF muscle for more than 30 minutes after the end of stimulation. There was no significant change in MEP log-amplitude for the BF muscle across the different time measures (Friedman's test, $\mathrm{p}=.28$ ).

In order to test if the absence of modulation observed for the BF muscle can be attributed to a subgroup effect, the analyses for the RF muscles were performed on the six participants submitted to the BF analyses. The Friedman's and Wilcoxon Signed-Rank tests revealed the same pattern of results obtained previously for the RF (i.e. decreased MEP log-amplitude at 60,75 and 90 minutes when compared to baseline; all p-values < $.05)$, hence arguing against the possibility that the absence of significant results obtained for the BF could be attributed to a subgroup effect or to a lack of power related to the smaller sample size. 


\section{Discussion}

In the present study, we investigated how the corticomotor excitability of thigh muscles is modulated following prolonged repetitive afferent electrical stimulation. We observed a decrease in corticospinal excitability after 60 minutes of stimulation for the RF muscle. This modulation was maintained 30 minutes after the end of stimulation and was specific to the RF, as suggested by the absence of significant changes for the BF muscle.

The present findings are coherent with the results of Mima et al. (10) who demonstrated that 30 minutes of high-frequency electrical stimulation applied on the thenar eminence of the hand decreased MEP amplitude. However, our results contrasts with the observations of Heroux et al. (13) who reported increased MEP responses after 30 minutes of high-frequency electrical stimulation applied on the anterior thigh. The differences between our results and the results of Heroux et al. (13) suggest that the modulations induced on lower-limb muscles by high-frequency stimulation depend on the duration of the stimulation. The influence of stimulation duration on corticomotor modulation was previously reported by Fraser and colleagues (11). In their experiment, Fraser et al. showed that the increased MEP responses noted for pharyngeal muscles following 10 minutes of low-frequency stimulation were no longer present when the stimulation was applied for 20 minutes. Taken together, our results and the results of Heroux et al. confirm and extend the observations of Fraser et al. by showing that stimulation duration can not only affect the magnitude of the modulation but also its direction.

\section{Absence of modulation for the biceps femoris}

Previous TMS studies have shown that the modulations induced by repetitive electrical nerve stimulation are specific to the stimulated region $(8,9,12)$. In their study, Hamdy et al. (9) noted that pharyngeal stimulation produced opposite changes in 
pharyngeal and esophageal MEP responses (i.e. increased responses for the pharyngeal muscle and decreased responses for the esophageal muscle). Even though they did not observe this opposite pattern of modulation, Ridding et al. (8) and Kaeling-Lang et al. (12) observed that the changes induced by electrical nerve stimulation were circumscribed to the region stimulated, with MEP changes occurring only in the muscles innervated by the stimulated nerve. The absence of MEP changes noted in the present study for the BF is consistent with these findings.

\section{Clinical applications and recommendations}

From a clinical perspective, the present results give credence to the use of electrical stimulation as a tool to promote neural plasticity. Although the long-term effects of electrical stimulation have not been investigated in the present study, it is reasonable to think that repeated sessions of afferent electrical stimulation could induce long-lasting changes in the central nervous system. Supporting this idea are the recent observations of Meesen and colleagues who demonstrated (1) that repeated daily applications of TENS over the hand area of healthy subjects increased the cortical motor representation of the ipsilateral hand and forearm muscles (16) and (2) that repeated use of TENS in a population of patients with multiple sclerosis produced a substantial increase in tactile sensitivity which lasted for more than three weeks following the intervention (17). Altogether, these findings substantiate the idea according to which repeated TENS applications can produce long-term changes and promote functional recovery.

Apart from its potential long-term effects, electrical stimulation might also be a valuable tool for health care professionals who wish to temporarily facilitate or inhibit movement during therapy. The use of TENS to reduce unwanted muscle contractions has previously been suggested by Levin \& Hui-Chan (18) who reported that repeated applications of high-frequency TENS over the common peroneal nerve decreased the spasticity and increased the voluntary motor functions in hemiplegic patients. Positive 
results have also been obtained by Foley-Nolan et al. (19) and Tinazzi et al. (20, 21) for focal hand dystonia. By documenting the corticospinal changes associated with high and low-frequency TENS protocols, TMS studies extend these clinical findings by showing : (1) that electrical stimulation can inhibit but also facilitate motor responses and (2) that these effects appear to rely mainly on changes occurring at the central level.

Importantly, the modulations induced by electrical stimulation appear to be highly dependent upon the intensity, frequency and duration of the stimulation (8-13). Hence, for clinicians who wish to facilitate motor responses, the use of low-frequency simulation protocols could be preferable. Alternately, when the goal is to inhibit unwanted muscle contractions and decrease spasticity, clinicians should prefer high-frequency stimulation protocols and keep in mind that longer stimulation periods ( $\sim 60$ minutes) seem to be necessary for lower-limb muscles. Yet, it is important to underline that the present study (as the vast majority of previous studies investigating the effect of electrical stimulation on corticospinal excitability) has been conducted in healthy subjects. As the response to electrical stimulation might be different in patients with neurological disorders, more research is needed in these populations prior to reaching definite conclusions on the clinical value of this method of facilitating or inhibiting motor responses.

\section{Limits}

Previous studies on the effect of electrical stimulation protocols applied over hand muscles have shown that the MEP modulations induced by electrical stimulation were largely due to changes occurring at the cortical level $(8,10)$. In agreement with these observations, Goulet et al. (22) observed that a high-frequency TENS protocol applied over the common peroneal nerve did not affect the amplitude of the H-reflex response. Although these observations may suggest that the pattern of MEP modulation seen in the present study is probably due to changes occurring at the cortical rather than spinal level, we cannot exclude this latter possibility, since no spinal excitability measures were taken. 
Future studies including both TMS and spinal measures are warranted before any definite conclusion can be made regarding the site of modulation.

Another important limitation that must be acknowledged concerns the differences between upper and lower-limb MEP responses evoked by TMS. Previous reports have suggested that the stimulation of the lower-limb motor cortex area with TMS preferentially produced D waves $(23,24)$, an observation which contrasted with the results of upper-limb studies showing that the application of TMS over the hand area of the motor cortex mainly produced I waves $(25,26)$. More recently, the selective implication of D waves in MEP responses evoked in lower-limb muscles were challenged by several authors who demonstrated that the amount of I waves were more important than originally postulated (27-29). Today, it is generally thought that TMS applied over the lower and upper extremity area of the motor cortex both preferentially activate I waves, although this preferential activation appears to be less consistent for the lowerextremity (29).

Finally, we must remember that the research paradigm used in the present study only involved one limb region (i.e. the anterior thigh). Although it might be tempting to generalize the results obtained in the present study to the entire lower-limb, we must remember that the effect of prolonged high-frequency stimulation may vary depending on the region that is stimulated. Future research focusing on the effects of prolonged highfrequency stimulation on other limb regions is therefore necessary. 


\section{Conclusion}

In the present study, we have shown that a high-frequency electrical stimulation protocol applied on the anterior thigh area selectively decreases the corticomotor excitability of the quadriceps muscle. Although the exact mechanisms responsible for this modulation are unknown, the current observations constitute an eloquent example of neuroplastic changes induced by sensory stimulation in humans, and provide an interesting venue for clinicians who wish to temporarily inhibit motor responses in lowerlimb muscles. Of importance, the present results indicate that the inhibitory effect induced by a 60 -minute high-frequency protocol can last for more than 30 minutes after ceasing stimulation, giving rehabilitation specialists sufficient time to work on the desired motor task. 
Acknowledgments: The authors wish to thank Paul Gauthier, Patrick Lalonde, Lucie Léonard, Shane Maley, Guillaume Michaud and Jessica Wilson for their help with data collection.

\section{Conflict of interest}

The authors declare that they have no conflict of interest. 


\section{References}

1. Duffau H. Brain plasticity: From pathophysiological mechanisms to therapeutic applications. J Clin Neurosci 2006; 13:885-97.

2. Nudo RJ. Plasticity. NeuroRx 2006;3:420-7.

3. Stehno-Bittel L. Neuroplasticity. In: Lundy-Ekman L, editor. Neuroscience:

Fundamentals for Rehabilitation. Toronto: W.B. Saunders Company; 2007, p. 71-83.

4. Flor H, Diers M. Sensorimotor training and cortical reorganization.

NeuroRehabilitation 2009;25:19-27.

5. Jang SH, Kim YH, Cho SH, Lee JH, Park JW, Kwon YH. Cortical reorganization induced by task-oriented training in chronic hemiplegic stroke patients. Neuroreport 2003; $14: 137-41$.

6. Sawaki L, Butler AJ, Leng X, Wassenaar PA, Mohammad YM, Blanton S, et al. Constraint-induced movement therapy results in increased motor map area in subjects 3 to 9 months after stroke. Neurorehabil Neural Repair 2008;22:505-13.

7. Recanzone GH, Allard TT, Jenkins WM, Merzenich MM. Receptive-field changes induced by peripheral nerve stimulation in SI of adult cats. J Neurophysiol 1990;63:121325 .

8. Ridding MC, Brouwer B, Miles TS, Pitcher JB, Thompson PD. Changes in muscle responses to stimulation of the motor cortex induced by peripheral nerve stimulation in human subjects. Exp Brain Res 2000;131:135-43.

9. Hamdy S, Rothwell JC, Aziz Q, Singh KD, Thompson DG. Long-term reorganization of human motor cortex driven by short-term sensory stimulation. Nat Neurosci

1998; $1: 64-8$.

10. Mima T, Oga T, Rothwell J, Satow T, Yamamoto J, Toma K, et al. Short-term highfrequency transcutaneous electrical nerve stimulation decreases human motor cortex excitability. Neurosci Lett 2004;355:85-8.

11. Fraser C, Power M, Hamdy S, Rothwell J, Hobday D, Hollander I, et al. Driving plasticity in human adult motor cortex is associated with improved motor function after brain injury. Neuron 2002;34:831-40.

12. Kaelin-Lang A, Luft AR, Sawaki L, Burstein AH, Sohn YH, Cohen LG. Modulation of human corticomotor excitability by somatosensory input. J Physiol 2002;540:623-33.

13. Heroux M, Tremblay F, Tremblay LE, Boisvenue M, Carrière M, Houston J. Differential effect of peripheral electrical stimulation and vibratory stimulation on corticomotor excitability in lower limb muscles. Physiotherapy Canada 2003;55:153-9. 
14. Mills KR, Nithi KA. Corticomotor threshold to magnetic stimulation: Normal values and repeatability. Muscle Nerve 1997;20:570-6.

15. Nielsen JF. Logarithmic distribution of amplitudes of compound muscle action potentials evoked by transcranial magnetic stimulation. J Clin Neurophysiol 1996; $13: 423-34$.

16. Meesen RL, Cuypers K, Rothwell JC, Swinnen SP, Levin O. The effect of long-term TENS on persistent neuroplastic changes in the human cerebral cortex. Hum Brain Mapp 2011;32:872-82.

17. Cuypers K, Levin O, Thijs H, Swinnen SP, Meesen RL. Long-term TENS treatment improves tactile sensitivity in MS patients. Neurorehabil Neural Repair 2010;24:420-7.

18. Levin MF, Hui-Chan CW. Relief of hemiparetic spasticity by TENS is associated with improvement in reflex and voluntary motor functions. Electroencephalogr Clin Neurophysiol 1992;85:131-42.

19. Foley-Nolan D, Kinirons M, Coughlan RJ, O'Connor P. Post whiplash dystonia well controlled by transcutaneous electrical nervous stimulation (TENS): Case report. J Trauma 1990;30:909-10.

20. Tinazzi M, Farina S, Bhatia K, Fiaschi A, Moretto G, Bertolasi L, et al. TENS for the treatment of writer's cramp dystonia: A randomized, placebo-controlled study. Neurology 2005; $64: 1946-8$.

21. Tinazzi M, Zarattini S, Valeriani M, Stanzani C, Moretto G, Smania N, et al. Effects of transcutaneous electrical nerve stimulation on motor cortex excitability in writer's cramp: Neurophysiological and clinical correlations. Mov Disord 2006;21:1908-13.

22. Goulet C, Arsenault AB, Levin MF, Bourbonnais D, Lepage Y. Absence of consistent effects of repetitive transcutaneous electrical stimulation on soleus H-reflex in normal subjects. Arch Phys Med Rehabil 1994;75:1132-6.

23. Terao Y, Ugawa Y, Sakai K, Uesaka Y, Kohara N, Kanazawa I. Transcranial stimulation of the leg area of the motor cortex in humans. Acta Neurol Scand 1994;89:378-83.

24. Priori A, Bertolasi L, Dressler D, Rothwell JC, Day BL, Thompson PD, et al. Transcranial electric and magnetic stimulation of the leg area of the human motor cortex: Single motor unit and surface EMG responses in the tibialis anterior muscle. Electroencephalogr Clin Neurophysiol 1993;89:131-7.

25. Day BL, Dressler D, Maertens de Noordhout A, Marsden CD, Nakashima K, Rothwell JC, et al. Electric and magnetic stimulation of human motor cortex: Surface EMG and single motor unit responses. J Physiol 1989;412:449-73. 
26. Amassian VE, Quirk GJ, Stewart M. A comparison of corticospinal activation by magnetic coil and electrical stimulation of monkey motor cortex. Electroencephalogr Clin Neurophysiol 1990;77:390-401.

27. Houlden DA, Schwartz ML, Tator CH, Ashby P, MacKay WA. Spinal cord-evoked potentials and muscle responses evoked by transcranial magnetic stimulation in 10 awake human subjects. J Neurosci 1999;19:1855-62.

28. Terao Y, Ugawa Y, Hanajima R, Machii K, Furubayashi T, Mochizuki H, et al. Predominant activation of I1-waves from the leg motor area by transcranial magnetic stimulation. Brain Res 2000;859:137-46.

29. Terao Y, Ugawa Y. Basic mechanisms of TMS. J Clin Neurophysiol 2002;19:322-43. 


\section{Figure Caption}

Figure1. Comparison of the mean variations in MEP amplitudes for (a) the rectus femoris and (b) the biceps femoris. Compared to baseline (T0), MEP amplitude for the rectus decreased after 60 minutes of electrical stimulation. The reduction in MEP amplitude was still present 15 and 30 minutes after the end of the electrical stimulation protocol. There was no change in MEP amplitude after TENS for the biceps femoris $(* p<.05)$.

A

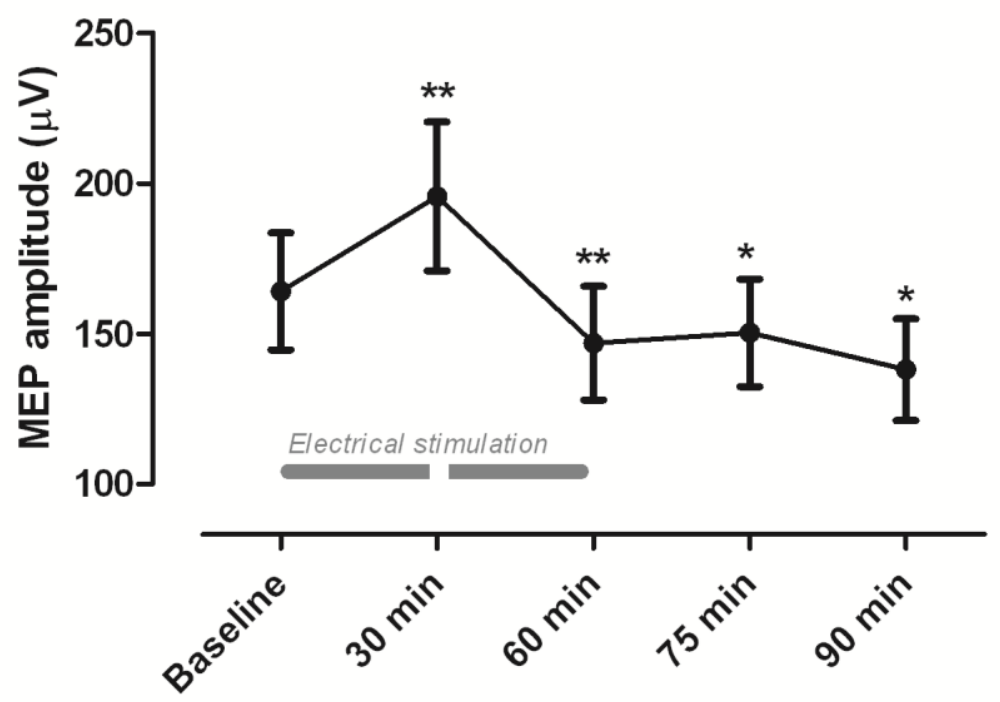

B

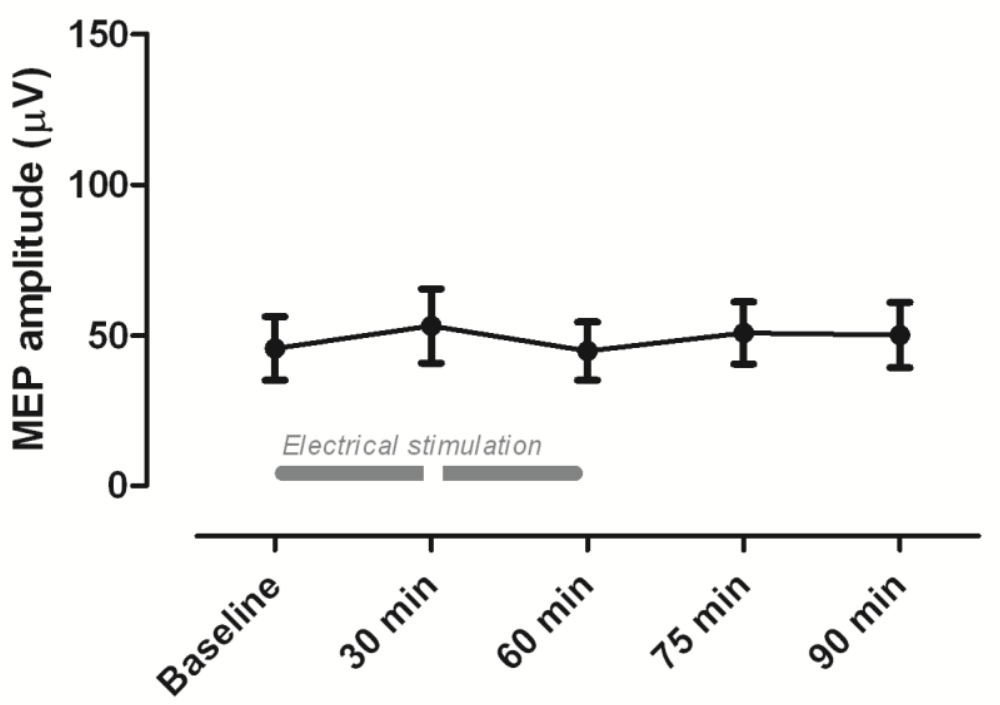

